\title{
Palliation of malignant ascites with a Tenckhoff catheter
}

\author{
D A LOMAS, P J W WALLIS, R A STOCKLEY \\ From the General Hospital, Birmingham
}

ABSTRACT To avoid multiple paracenteses, a Tenckhoff catheter was used successfully to drain the ascitic fluid in a patient with peritoneal mesothelioma.

Malignant mesothelioma affects 1-2 individuals per million population each year. Twenty per cent of the tumours arise within the peritoneal cavity and subsequent ascites may provide a major management problem. We report the use of a Tenckhoff catheter for palliation of continued symptoms.

\section{Case report}

A 60 year old man presented with a one month history of increasing abdominal swelling and a painful swollen right scrotum. He had a non-productive cough and mild breathlessness on exertion. He was a lifelong non-smoker and consumed alcohol in moderation. He was receiving no medication. In 1959 he had had a left inguinal hernia repair and orchidectomy and, although the histological appearances raised the possibility of a teratoma, this was not confirmed at follow up. Since leaving school he had been employed in the bus and train construction industry and for 20 years had "sprayed" blue asbestos on to the bodywork of these vehicles. His exposure to asbestos had been heavy and he had had to comb asbestos fibres from his hair at the end of each day.

On examination he was overweight and tachypnoeic. Examination of the cardiovascular and respiratory systems showed nothing remarkable but examination of the abdomen revealed dilated superficial veins, ascites, a tense right scrotal swelling, and a firm deposit around the umbilicus.

The full blood count and results of renal and liver function tests were all normal. An abdominal ultrasound scan confirmed gross ascites and showed that the right scrotal swelling consisted of fluid with a normal testis. The chest radiograph showed pleural thickening with areas of calcification consistent with pleural plaques due to asbestos exposure. A Tru-cut biopsy specimen of the umbilical deposit and the cytological appearance of the ascitic fluid were consistent with a diagnosis of malignant mesothelioma. He was initially treated with diuretics but this failed to control his gross ascites. To avoid multiple paracenteses a Tenckhoff catheter was inserted and the ascitic fluid drained. Eighteen litres of fluid were drained over the first seven days, with rapid improvement in his symptoms and reduction of his scrotal

Address for reprint requests: Dr D A Lomas, General Hospital, Birmingham B4 6NH.

Accepted 20 July 1989 swelling. He was discharged with instructions to drain 1 litre $\overrightarrow{-}$ of fluid each day.

Over the next three months he gradually deteriorated, with anorexia, weight loss, and a bloated feeling. He developed ${ }^{x}$ peripheral oedema that responded to elevation of the legs. The catheter continued to drain 1 litre a day, controlling theascites and the abdominal discomfort. The protein content of the ascitic fluid was $22 \mathrm{~g} / 1$ with an albumin content of $8 \mathrm{~g} / 1 \mathrm{~N}$ The serum albumin fell from $28 \mathrm{~g} / \mathrm{l}$ at the time of insertion of the catheter to $18 \mathrm{~g} / \mathrm{l}$. His condition deteriorated further and he died three months after presentation. Necropsy disclosedbilateral plaques in the costal and diaphragmatic parieta? pleura. There was diffuse mesothelioma affecting the parieta $\bar{b}^{\circ}$ and visceral peritoneum, though only a small quantity of olood stained fluid was present in the peritoneal cavity.

\section{Discussion}

Some $10-20 \%$ of malignant mesotheliomas arise in the peritoneal cavity. ${ }^{1}$ Radical treatment with chemotherapy and radiotherapy is of limited value ${ }^{2}$ and often the emphasis is on palliation of symptoms. The ascites that accompanie品 peritoneal mesothelioma may respond to diuretic treatmen $\underline{D}$ coupled with salt and water restriction; if this fails the patien may be considered for insertion of a LeVeen peritoneovenous shunt. ${ }^{3}$ The shunt may, however, be associated with many complications, ${ }^{4}$ such as disseminated intravascular coagula tion, pulmonary oedema, sepsis, pulmonary emboli, venous thrombosis, bowel perforation, tumour embolisation, and tumour dissemination.' We report the successful use of a Tenckhoff catheter to relieve ascites that had failed to respond to more conservative management. The removal op ascites may have contributed to the fall in serum albumip although the patient was anorexic during this period and wa\& in the terminal stages of his illness. Despite the fall in albumin he had only mild peripheral oedema, which responded to elevation of the legs. We would recommend this treatment for palliation of patients with peritoneal malignant meso thelioma and intractable ascites.

\section{References}

1 Legha SS, Muggia FM. Pleural mesothelioma: clinical feature and therapeutic implications. Acta Intern Med 1977;87:613-2 K.

2 Lerner HJ, Schoenfield DA, Martin A, Falkson G, Borden E을 Malignant mesothelioma. Cancer 1983;53:1981-5.

3 Plaus WJ. Peritoneal mesothelioma. Arch Surg 1988;123:763-6. \&

4 Cheung DK, Raaf JH. Selection of patients with malignant ascites for a peritoneovenous shunt. Cancer 1982;50:1204-9.

5 Nervino HE, Gebhardt FC. Peritoneovenous shunt for intractabl malignant ascites. A single case report of metastatic peritoneas mesothelioma implanted via LeVeen Shunt. Cance $1984 ; 54: 2231-3$ 